\title{
RF Energy Harvesting for Low Power Devices
}

\author{
D. Srinivasulu Reddy \\ Professor, Department of ECE, S V College of Engineering, Tirupati, A.P, India
}

\begin{abstract}
Radio Frequency is abundandly available in an outdoor environment. The number of existing RF bands is impulsive. The RF circuit has to harvest all the potential RF energy present and cannot be premeditated for a specific RF tone. The designed rectenna adds powers coming from an limiteless number of sub frequency bands. The designed rectenna output voltage increase with the number of RF bands. As an application, a Radio Frequency band rectenna is designed. The system uses PIFA (Planar Inverted F Antenna) to harvests energy from GSM900 (Global System for Mobile Communications), GSM1800, UMTS (Universal Mobile Telecommunication Service) and WiFi bands simultaneously. The planar inverted F antenna is popular for portable devices because of its low profile, small size, and built-in structure. The radiated power from the antenna is given as input to the rectifier. The schottky diode is used for rectification. Inorder to get maximum power to the load the $\mathrm{T}$ matching is used to get an proper impedance matching. The seven stage voltage doubler of HSMS 2850 is introduced in the circuit in order to get maximum voltage to supply the low power devices.
\end{abstract}

KEYWORDS: Multiband, rectenna, RF energy harvesting, Planar inverted F antenna

\section{INTRODUCTION}

Radio frequency energy is emanated by sources that generate high electromagnetic fields such as TV signals, wireless radio networks and cell towers, but through using a power generating circuit linked to a receiving antenna. The free flowing energy can be captured and translated into usable DC voltage. There are number of alternative ways to extract energy from the surroundings and convert it into electrical energy for strengthen low power electronic circuits directly or gather it for later on use. One such energy is from radio frequency. RF Energy harvesting from the ambient will have an significant role in the future microelectronic circuits. The energy is freely available in space, Complementing the stumpy power sources used to boost the low power electronic devices, as an purpose to green technology. Radio Frequency energy harvesting from ambient sources have great possible to impact on the cellular phones and portable electronic devices. This model needs an

proficient antenna along with a circuit capable of converting RF signals into DC voltage, to replace the need for batteries.

Recent advances in RF energy-harvesting technology have made self-sustainable devices feasible. The major concern for these devices is typically the battery life and replacement. Applying RF energy-harvesting technique to them can significantly extend the battery life and sometimes even avoid the need for a battery. The ambient RF power is a good potential for the energy supply as it is widely broadcast from numerous reliable electromagnetic resources. However, since the power density of the ambient RF power is extremely small, it is very challenging to design RF energy-harvesting systems with satisfying RF-to-dc power conversion efficiencies (PCEs).the decrease of electronic components consumption has led to the development of wireless devices. Unlike most energy sources, the EM or RF energy sources are continuously available. When multiple RF energy sources are available, the amount of energy harvested can be increased if the system is designed to work over a wide frequency band. From theory analysis supported by simulation designed to cover the GSM900 (Global System for Mobile Communications), GSM1800, UMTS2100, and WiFi bands. The GSM900 entire band is from 876 to $959 \mathrm{MHz}$ including UpLink (UL) and DownLink (DL) modes. The GSM1800 is from 1710 to1880 MHz (including UL and DL modes). The UMTS band is from 1920 to $2170 \mathrm{MHz}$ (including UL and DL modes), and finally the WiFi band is from 2.41 to $2.48 \mathrm{GHz}$. The RF harvester measurements show that the proposed stacked multi-band RF harvester is able to supply typical electronic devices. It can charge low power devices such as watch, calculator (10microWatt), Hearing aid, temperature sensors (100 microWatt), Active RFID (1 mW).

\section{RELATED WORK}

Zied Harouni developed a hybrid sensitive rectenna system at $2.45 \mathrm{GHz}$. They first optimized and validated a zero-bias microwave sensitive rectifier using commercial schottky diodes. This system was able to have a high sensitivity at low power levels. The commercial diode HSMS2820 was selected for the rectifier circuit[1].[2]Four 
harvesters designed to cover four frequency bands within the ultrahigh frequency $(0.3-3 \mathrm{GHz})$ part of the frequency spectrum. Samuel S. B. Hong, Rosdiazli Ibrahim developed that Battery life could be extended by scavenging surrounding Wi-Fi signals using rectenna architecture which consists of a receiving antenna attached to a rectifying circuit[3]. A seven stage Cockroft-Walton voltage multiplier optimized for low input power is proposed.Filters are needed to attenuate high frequency harmonics present in the RF signal or generated by the highly nonlinear rectification process.CMOS based voltage doubler circuit is used to achieve $160 \%$ increase in output power over traditional circuits at $0 \mathrm{dBm}$ input power[7].[10]Multistage Schottky rectifier antenna (rectenna) array circuit is proposed for high energy conversion efficiency to improve battery life and portability.

\section{PROPOSED SYSTEM}

This section provides the detailed description of the proposed system. The system design is shown in Figure 1.

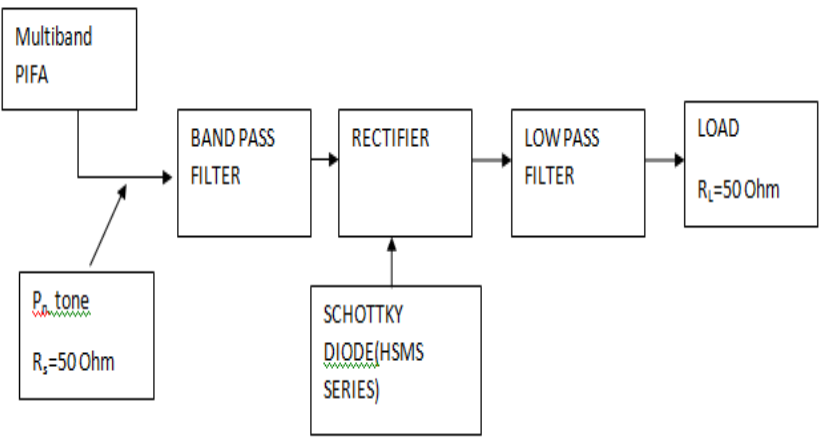

Figure 1. Proposed Rectenna Block Diagram

It deals with the design of the following modules: i. an antenna for maximizing RF signal, ii. a suitable matching network to match the complex load impedance to the antenna impedance.iii. rectifier for rf to dc conversion.

\section{A.}

PIFA DESIGN

This antenna design is used to cover

GSM900,GSM1800,UMTS2100,WiFi bands. This problem of covering multiple bands with a single antenna structure is the need of today's mobile devices and it can be achieved by using low profile antenna structures like PIFA. FR4 substrate is used as dielectric material with loss tangent, $\delta=0.02$; dielectric constant, $\varepsilon r=4.4$ and substrate height, $\mathrm{h}=1.6 \mathrm{~mm}$. A coaxial cable is used to feed the antenna from the backside of PCB where ground plane lies. Air is used as dielectric between FR4 substrate and top radiating patch. The proposed antenna consists of planar radiating element, two rectangular slots on the ground plane, a shorting plate, coaxial feed and a ground plane. The dimensions of the rectangular patch of the antenna are $25 \times 15 \times 3.8 \mathrm{~mm}^{3}$ and that of ground plane are $70 \times 40 \times 3.8 \mathrm{~mm}^{3}$. The height of PIFA selected is $2.2 \mathrm{~mm}$ from the FR-4 substrate and $3.8 \mathrm{~mm}$ from the ground plane.

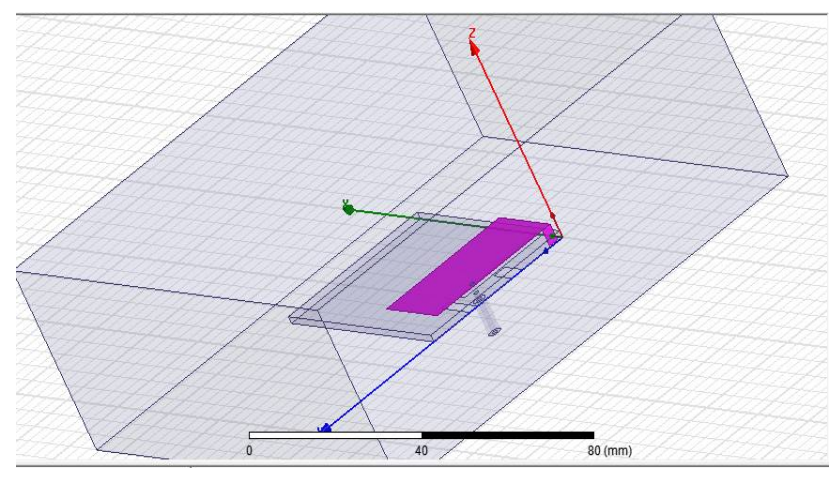

Figure 2. 3D View of PIFA 
International Journal of Advanced Research in Electrical, Electronics and Instrumentation Engineering Vol. 1, Issue 1, July 2012

\section{B. RECTIFIER IMPACT ON RF ENERGY HARVESTER}

The ambient RF energy harvester is to convert the RF energy received from RF ambient sources into dc power. Such harvesters are called rectenna, i.e.,rectifying antenna. A typical rectenna circuit consists of a receiving antenna followed by an RF band pass filter, a rectifier, a low-pass filter and a load. The filter functionality is to match the antenna impedance and the conjugate impedance of the rectifier input. Further more, the rectifier input impedance varies as a function of the frequency and the incident power. The antenna impedance may also vary as a function of the frequency.

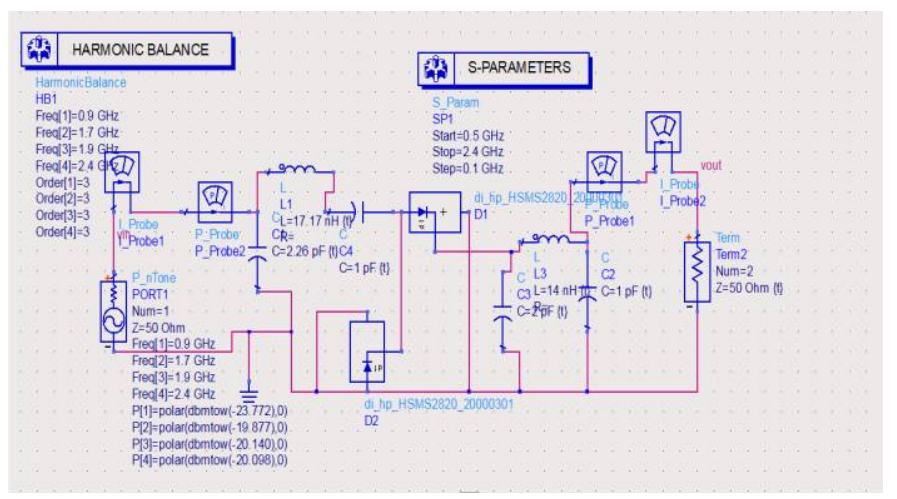

Figure 3. Circuit Diagram of Rectifier

The power received from the antenna are given as input to the rectifier.To match the antenna impedance with the rectifier impedance $\mathrm{T}$ matching is used. Band pass filter is used as an pre rectification process inorder to remove higher order harmonics. After the voltage drop at the diode, the signal is passed through the low pass filter. The output of the rectifier contains DC components as well as AC components. Filters are used to minimise the undesirable AC, that is ripple leaving only the DC component to

appear at the output. For that LC or L section filter is used. The simplest of these circuits are a form of rectifier which take an AC voltage as input and outputs a doubled DC voltage. The switching elements are simple diodes and they are driven to switch state merely by the alternating voltage of the input. by an load of $50 \mathrm{ohm}$. The output voltage of the voltage doubler is shown in Figure 6.

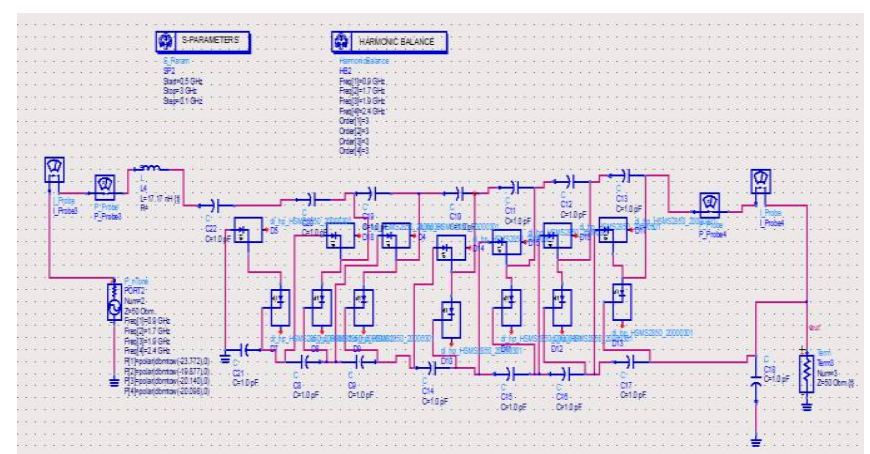

Figure 4. Circuit Diagram of Voltage Doubler Circuit

The seven stage voltage doubler is used to get an high output DC voltage as shown in Figure 4. The power received from the antenna are given as input to the rectifier. The schottky diode series of HSMS 2850 is used along with an capacitor and then terminated with an load of $50 \mathrm{Ohm}$.

\section{SIMULATION AND RESULTS}

\section{A. RECTIFIER OUTPUT}

The voltage at the input and the output of the Figure 6. Output Voltage

\section{B. ANTENNA OUTPUT}

Return loss is a measure of the effectiveness of power delivery from a transmission line to a load such as an antenna. If the power incident on the antenna under test(AUT) is Pin and the power reflected back to the source is Pref, the degree of mismatch between the incident and reflected power in the travelling wave is given by the 
ratio Pin/Pref. The higher this power ratio, the better the load and line are matched.

Return loss is expressed in $\mathrm{dB}$.

In order to over come it, the voltage doubler is used as an rectifier to get an high output DC voltage. The Pn tone is used to receive power from multibands. The four frequencies are specified by using an Pn tone. It is then passed through an seven stage voltage doubler which constitutes of an HSMS 2850 diode and thus it is terminated The return loss is measured for PIFA antenna as shown in Figure 7.Considering it as an multiband,for GSM 1800,WiFi it has high return loss. For these bands the gain of an antenna is positive and thus the radiation efficiency is also above $90 \%$ for all bands.

\section{ANTENNA PARAMETERS}

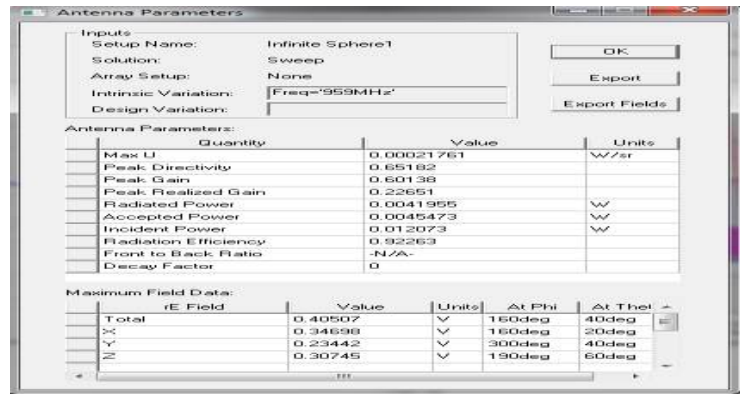

Figure 8. Antenna Parameters for GSM 900 Band

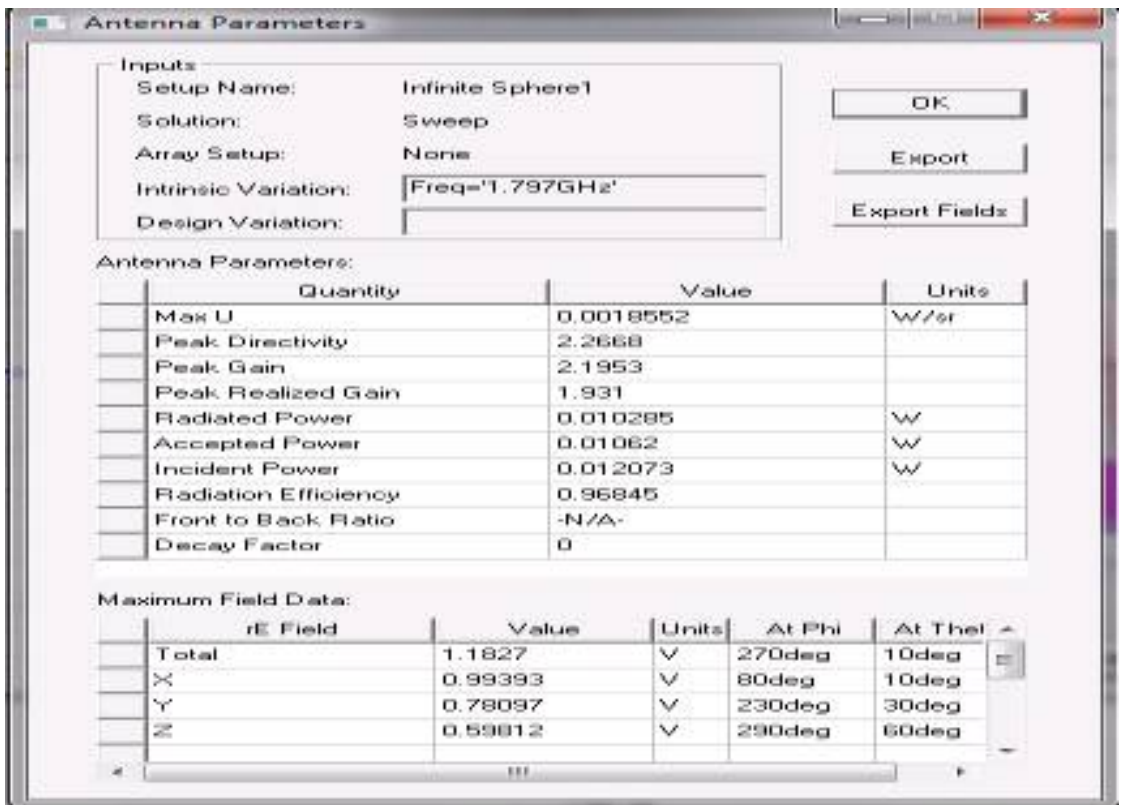

Thus ,the radiation efficiency is large for WiFi band as shown in Figure 11. compared to other band of frequencies. Table 1. Gain and Efficiency of PIFA for Different Bands

\begin{tabular}{|l|l|l|l|}
\hline Bands & $\begin{array}{l}\text { Freq } \\
(\mathrm{GHz})\end{array}$ & Gain & $\square$ \\
\hline GSM 900 & 0.959 & 0.601 & 92.00 \\
\hline GSM 1800 & 1.79 & 2.1953 & 96.84 \\
\hline UMTS 2100 & 1.91 & 2.682 & 93.52 \\
\hline WiFi & 2.41 & 6.493 & 97 \\
\hline
\end{tabular}

The gain and efficiency of different bands are as shown in Table 1. thus the radiation efficiency of an antenna is of 
above $90 \%$ and the gain obtained is also positive for all bands.

\section{EFFICIENCY OF RECTENNA}

The efficiency is calculated using this formula

Figure 9. Antenna Parameters for GSM 1800 Band

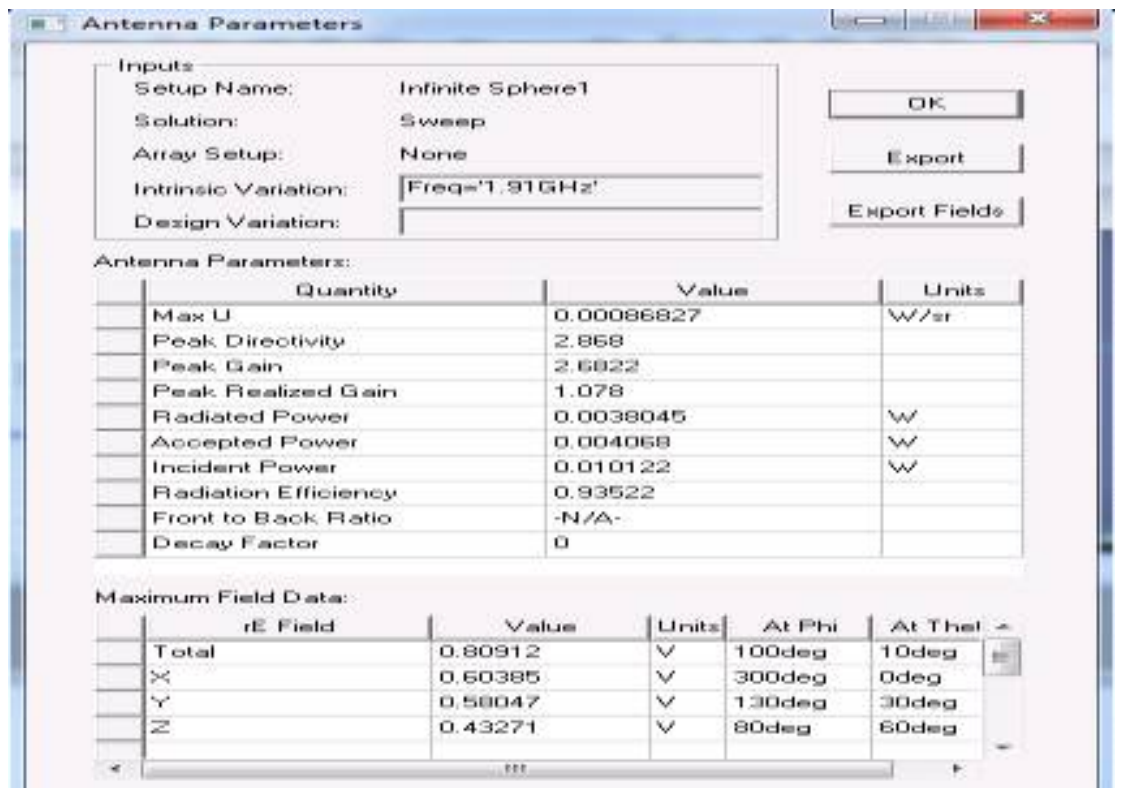

Table 2. Efficiency of the Rectenna.

\begin{tabular}{|l|l|l|l|l|}
\hline Frequency & $\begin{array}{l}\text { Input } \\
\text { power } \\
\text { (W) }\end{array}$ & $\begin{array}{l}\text { Resistance } \\
\text { (ohms) }\end{array}$ & $\begin{array}{l}\text { Voltage } \\
\text { (V) }\end{array}$ & $\begin{array}{l}\text { Efficiency } \\
(\%)\end{array}$ \\
\hline $0.9 \mathrm{GHz}$ & 0.275 & 50 & 3.279 & 76.936 \\
$1.7 \mathrm{GHz}$ & & & & \\
$1.9 \mathrm{GHz}$ & & & & \\
$2.4 \mathrm{GHz}$ & & & & \\
\hline
\end{tabular}

The output DC voltage is measured across the load resistance. The voltage across the load VL is $3.279 \mathrm{~V}$. The efficiency of the rectenna is $76.936 \%$ for the given input power level as shown in Table 2 .

\section{CONCLUSION AND FUTURE WORK}

The over all rectenna system is carried out by the combination of an antenna and rectifier circuit. The power of an antenna is incorporated into the rectifier circuit. The rectifier converts the AC energy given by the antenna into the DC power. The impedance matching between antenna and the rectifying circuit always affects the overall rectifying efficiency. Here T-matching 
circuit and LC filter section is provided for that purpose. The output DC voltage is measured across the load resistance. The voltage across the load $\mathrm{VL}_{\mathrm{L}}$ is 3.279 V.The efficiency of the rectenna is $76.936 \%$ for the given input power level. When the input power increases the rectifying efficiency also increases. Thus radiated power from the antenna should be improved for the increased efficiency of the rectenna. The antenna should be further optimized to get high return loss and high gain.Another type of rectifier circuit such as super capacitor model is to be used which replaces the resistive load with the capacitor load.

\section{REFERENCES}

[1] Zied Harouni, Lotfi Osman, Ali Gharsallah, "Efficient 2.45Ghz Rectenna Design with High Harmonics Rejection for Wireless Power Transmission" in IJCSI, vol. 7, Issue 5,pp.418-423, September 2010.

[2] Manuel Pinuela, Paul D. Mitcheson, Stepan Lucyszyn, “Ambient RF Energy Harvesting in Urban and Semi-Urban Environments"in IEEE transactions on microwave theory and techniques, vol. 61, no. 7, July 2013.

[3] Samuel S.B.Hong, Rosdiazli Ibrahim, Mohd H.Md.Khir,Mohammad A. Zakariya and Hanita Daud, "WI FI Energy harvester for low power RFID applications"Progress in Electromagnetic Research Research C, Vol. 40, 69-81, 2013.

[4] K.Niotaki et al. "A compact dual-band rectenna using slot-loaded dual band folded dipole antenna," IEEE Antennas Wireless Propag. Lett. ,vol.12,pp.1634-1637,2013.

[5] U.Olgun,C.-C.Chen,and J.L.Volakis,"Design of an efficient ambient WiFi energy harvesting system," IET Microw., Antennas, Propag. ,vol.6,no.11,pp.1200-1206,2012.

[6] Haruichi Kanaya,Shoichiro Tsukamoto, akuya Hirabaru, "Energy Harvesting Circuit on a One-Sided Directional Flexible Antenna" IEEE microwave and wireless components letters, vol. 23, no. 3, MARCH 2013.

[7] Hamid Jabbar,Taikyeong Ted. Jeong "RF Energy Harvesting System and Circuits for Charging of Mobile Devices" IEEE 2010.

[8] W.huang,B.zhang,X.chen,K.M.huang,“Study of S band rectenna array for wireless microwave power transmission”Progr. Electromagn Res.,vol 135,pp 747-758,2013.

[9] Bo Li,Xi Shao, Negin Shahshahan,Neil Goldsman, Thomas Salter, and George M. Metze“An Antenna Co-Design Dual Band RF Energy Harvester" IEEE transaction on circuits and systems-I: regular papers, vol. 60, no. 12, December 2013.

[10] J.Michael Barcak,Hakan P.Partal,'Efficient RF energy harvesting by using multiband microstrip antenna arrays with multistage rectifiers'IEEE subthreshold microelectronic conference 2012. 